\title{
La recherche comme projet à rebours
}

Research as Retro-Design

\section{Denis Delbaere et Bernadette Blanchon}

\section{(2) OpenEdition}

Journals

Édition électronique

URL : http://journals.openedition.org/crau/554

DOI : $10.4000 /$ crau. 554

ISSN : 2547-5746

\section{Éditeur}

Éditions du patrimoine

\section{Édition imprimée}

Date de publication : 1 novembre 2012

Pagination : 103-111

ISBN : 978-2-7577-0108-9

ISSN : 1296-4077

Référence électronique

Denis Delbaere et Bernadette Blanchon, "La recherche comme projet à rebours ", Les Cahiers de la recherche architecturale et urbaine [En ligne], 26/27 | 2012, mis en ligne le 01 novembre 2017, consulté le 03 mai 2019. URL : http://journals.openedition.org/crau/554 ; DOI : 10.4000/crau.554 
La recherche trouvera sa place dans les écoles de projet, qu'il soit d'architecture ou de paysage, en fonction de la capacité à construire de solides passerelles entre pensée réflexive et action. Le présent article relate une expérience de recherche proposant une telle interface. La recherche de la qualité paysagère de près de 300 grands ensembles français a mis en évidence, par un travail de terrain, et en archives, les projets de paysage latents qui façonnent certains de ces ensembles urbains. Le chercheur devient ici, d'une certaine manière, l'auteur d'un projet qu'on ne peut apercevoir que rétrospectivement.

\section{La recherche comme projet à rebours}

DENIS DELBAERE

BERNADETTE BLANCHON

" C'est une autre dimension du paysage qui m'intéresse, celle de l'antériorité des sites sur lesquels on nous demande de projeter. Tous les espaces que l'on veut modifier, aussi déficients soient-ils, ont déjà fait l'objet de nombreuses affectations, de plusieurs aménagements, dont ils gardent certains témoignages. Ils sont enchâssés dans un ensemble plus vaste d'espaces mitoyens, avec lesquels ils entretiennent de multiples relations; c'est ce que j'appelle leurs divers horizons. L'histoire, toujours très longue, qui leur a donné leur statut actuel, m'interdit de penser qu'ils sont tout à fait disponibles»

(Michel Corajoud ${ }^{1}$ ).

Le statut de la recherche au sein des disciplines de l'architecture, de I'urbanisme et du paysage, interroge leur sens même. En effet, comment ces disciplines, qui sont intimement liées via l'activité de projet au champ de l'action et de la transformation du monde, pourraient-elles être pensées également comme des disciplines de l'observation, de la

1. «Paysage : on aimerait tant photographier un paysage de dos", débat sur le paysage entre Alexandre Chemetoff, Michel Corajoud, Michel Desvigne et Bernard Huet, L'Architecture d'Aujourd'hui n 262, avril 1989, p. 33. 
conceptualisation et de la déduction causale? Personne ne remet en question la possibilité et l'utilité d'une recherche « sur » les disciplines du projet - recherches qui empruntent aux méthodes des diverses sciences humaines -, mais que peut-il en être d'une recherche "dans " ces disciplines, d'une recherche qui, du coup, prétendrait participer elle-même de l'activité de projet?

La démarche relatée illustre I'un des multiples biais par lesquels la recherche peut relever de la démarche de projet. Par un mode d'investigation de type historique, il s'est agi ici, en effet, de projeter, mais de projeter "à l'envers ». Le projet, dans le cas qui nous intéresse, n'est pas devant, mais derrière nous, et le travail du chercheur a précisément consisté à renouer avec ce projet antérieur, à le relire, en mobilisant les savoirs de I'historien mais aussi, en l'occurrence, du paysagiste. Et la redécouverte de ces projets oubliés, dont ne demeurent dans l'espace que quelques traces plus ou moins tangibles, débouche sur une forme d'action, en permettant de préserver et de redéployer les qualités des espaces considérés.

\section{Un « rétro-projet » pour les grands ensembles? Le paysage dans les ensembles urbains de logement en France de 1940 à 1980}

La commande dont cette recherche est issue porte sur la qualité paysagère des grands ensembles français. Depuis la création de l'Agence nationale pour la rénovation urbaine, des chantiers de remodelage d'une envergure nouvelle ont pris place en France. Sans préjuger de la pertinence de ces opérations, il a pu être largement constaté que certaines d'entre elles présentaient un caractère inutilement agressif à l'égard non seulement des bâtiments, mais aussi des espaces publics. Or, ce dernier aspect des grands ensembles - leur qualité paysagère -est généralement inaperçue et cette circonstance pose d'emblée une caractéristique du projet à rebours, ou "rétro-projet " tel que nous voulons l'illustrer : il porte sur un objet bien réel, mais peu, voire pas considéré. Le chercheur, en se penchant sur cet objet, le découvre un peu comme on peut dire d'un explorateur qu'il crée en l'inventant (au sens étymologique du terme, "invenire ", "trouver, inventer ") l'objet dont il traite. Cette manière de "faire projet " par l'« invention " d'un objet s'inscrit d'ailleurs profondément dans la nature même de l'objet paysage, puisque tout un pan de la théorie du paysage en France a pu affirmer que le paysage est, moins qu'un espace physique, une dimension, une qualité de l'espace perçu par un observateur ${ }^{2}$. De fait, la démarche de projet de paysage comporte presque toujours un préalable analytique, un état des lieux justement qualifié d'inventif par différents paysagistes $^{3}$, par lequel l'espace considéré accède, avant même sa transformation par le projet d'aménagement, au statut de paysage, véritable préalable à toute forme d'action efficace.

En 2008, la Direction générale des patrimoines a engagé trois recherches portant sur la qualité constructive, architecturale et paysagère de ces quartiers. Chargés du troisième volet, nous avons pu étudier 284 ensembles urbains de la période 1940-19804, en y recherchant une dimension paysagère susceptible d'être
2. Nous pensons ici notamment à la théorie de l'artialisation telle que formulée par Alain Roger, notamment dans son Court Traité du Paysage, Paris, Gallimard, 1997.

3. On doit à Bernard Lassus la formulation de la notion d' " analyse inventive " pour décrire ce processus. Michel Corajoud développe des propositions comparables dans "le projet de paysage : lettre aux étudiants 》 (dans Le jardinier, l'artiste et
I'ingénieur, Jean-Luc Brisson (dir), Paris, Les Éditions de l'Imprimeur, 2000).

4. Cette liste a été établie par Bruno Vayssière dans les années 1980 au sein de I'Institut français d'architecture, au terme de recherches bibliographiques à partir de la presse spécialisée d'époque. Bien qu'évidemment non exhaustive, elle a servi de base à cette première investigation systématique dans le paysage des grands ensembles français. 
valorisée et exploitée dans le cadre d'une restructuration plus ouverte ${ }^{5}$. II convient de remarquer d'emblée que l'intitulé de la recherche portait sur « le paysage dans les ensembles urbains de logement ", et non pas sur les « projets de paysage » qui auraient pu, au même titre que les projets d'architecture, y instiller une qualité particulière. L'énoncé pose immédiatement le paysage comme une qualité infuse des grands ensembles, et non pas comme l'effet d'une volonté consciente, dès leur création, de composer leurs espaces "libres » autour d'un certain nombre d'objectifs affichés de qualité. Ce " déni de projet » est révélateur de la reconnaissance encore très faible qui est accordée en France à la pensée paysagiste des grands ensembles, pensée pourtant majeure et qui a donné lieu à certaines réalisations essentielles. Pourtant, la trop fréquente indigence de la production courante résulte souvent d'une mise en œuvre trop partielle des projets de paysage, ou d'une absence de gestion adaptée. Quoi qu'il en soit, l'essentiel de la culture contemporaine du paysage trouve l'une de ses origines dans ces réalisations, comme le montrent nos contributions en la matière ${ }^{6}$. D'un autre côté, le fait que la recherche porte sur «le paysage » et non pas sur les « projets de paysage » présente l'avantage d'installer son objet dans un état de latence et d'indéfinition qui le dispose d'autant au travail d'invention tel que nous venons de le décrire. Pour nous, dès lors, le travail consistait à rechercher les opérations qui, d'une façon ou d'une autre, ont contribué à façonner positivement, activement et volontairement l'espace public de ces quartiers.

Mais comment faire ressurgir des projets qui, sauf exception, n'ont fait l'objet que d'une médiatisation très limitée ? En général, les grands ensembles n'ont pas fait l'objet d'un projet de paysage spécifique (cela reste le cas aujourd'hui, avec leur réhabilitation). Parfois, leurs architectes étaient eux-mêmes imprégnés de " culture paysagiste » et la qualité des espaces publics dans leurs projets s'en ressent (on pense ici à ces grands « architectes paysagistes » que furent Émile Aillaud et Jean Dubuisson, Robert Auzelle et Marcel Breuer ou encore Jean Fayeton et Fernand Pouillon). Parfois encore, le projet de paysage a été confié à un paysagiste qui agit dans l'ombre de l'architecte, mais dont le travail a été peu, voire pas publié ni conservé (c'est le cas de la plupart des projets de Jacques Simon, Jacques Sgard, Jean-Claude Saint-Maurice, etc.). Le plus souvent, en l'absence de paysagiste, c'est l'entrepreneur paysagiste lui-même qui joua le rôle du concepteur, d'autant plus facilement qu'il a fallu attendre les années 1970 pour que la discipline du paysage dispose d'un enseignement spécifique, clairement distinct de sa gangue horticole.

Dans ces conditions, le travail de recherche et d'analyse des archives projectuelles des quartiers étudiés ne peut, comme dans le cas d'une recherche historique "classique », suffire à faire ressurgir le projet étudié. II a donc fallu procéder de manière inverse : non pas partir des archives pour lire l'espace du grand ensemble actuel, mais lire avec les outils de la pensée paysagiste l'espace du grand ensemble pour en déduire les fondements du projet qui l'a mis en forme. Ces outils ont été mobilisés à trois échelles :

- l'échelle territoriale de l'insertion dans leur contexte géographique (topographique, hydraulique, végétal, viaire et urbain) ;

5. Bernadette Blanchon, Denis Delbaere, Jörn Garleff (dir.), Le paysage dans les ensembles urbains de logement en France de 1940 à 1980, rapport de recherche, école nationale supérieure de paysage de Versailles, Direction générale des patrimoines, ministère de la Culture et de la Communication, juin 2010. 
- l'échelle du plan de masse, des formes des espaces publics, de leur diversité et de leur mise en relation, déterminée par l'implantation des bâtiments ;

- enfin, l'échelle plus proche des pratiques et des formes de relations entre l'espace privé des logements et l'espace public.

Ces méthodes de lecture du projet depuis le lieu qu'il a investi sont aussi induites par le rapport particulier qu'entretient le projet de paysage avec le temps. Les écarts entre projet et réalisation, les aléas de la gestion, l'essence même de cet " art de prévoir l'imprévisible " rendent illusoire la recherche d'un état initial idéal qu'il s'agirait de restituer. II faut au contraire procéder "à rebours", lire le projet de paysage tel que le site nous le donne à voir et à vivre, comme une forme de cohérence toujours en train de se faire, au gré des projets, des restructurations, des décisions prises quotidiennement sur les formes et la gestion de l'espace public.

Chacun des 284 sites étudiés a ainsi fait l'objet d'une première analyse à travers des cartes géographiques, des vues aériennes, et des articles de la presse professionnelle d'époque. Nous avons ensuite retenu 60 quartiers qui ont fait l'objet d'investigations sur le terrain, relatées sous forme de reportages photographiques, de schémas et de coupes. Enfin, 9 sites remarquables ont fait l'objet d'une recherche approfondie en archives. Au final, la confrontation de ces différentes sources permet la comparaison entre les intentions initiales éventuellement mises à jour par les archives, et les effets du temps et des usages observés sur place.

\section{Méthode : débusquer les marqueurs du projet de paysage}

Ce que notre recherche met tout d'abord en évidence, c'est l'extrême diversité des situations paysagères, dont le classement typologique ne va pas sans poser problème. La classification la plus évidente reposerait sur les plans de masse qui sont à la base de l'urbanisme de cette période, et dont la diversité résulte en partie de l'évolution des politiques publiques du logement social et de la pensée architecturale de l'époque. Dans les années 1945-1955, les projets s'inscrivent dans la poursuite des règles de l'art urbain issues de l'entredeux-guerres et nourries par la formation de l'École des beaux-arts (La Butte Rouge à Châtenay-Malabry, Beaulieu à Saint-Étienne...). La figure du grand espace central y est dominante (Beaudouin à la Cité universitaire d'Antony ou à la cité Rotterdam à Strasbourg...) avant de laisser place à la suprématie de "l'ordre ouvert ». Les opérations expérimentales (le Shape à Saint-Germain-en-Laye, Bron-Parilly...) opèrent des assemblages de formes diverses faisant également appel à des savoirs faire antérieurs; alors que dans les opérations d'envergure (2500 à 5000 logements) qui suivent l'appel de l'abbé Pierre dominent les implantations en strates parallèles ou en trames orthogonales ainsi que quelques semis de tours ou de plots (Les Lochères à Sarcelles, les 4000 à La Courneuve). Les concepteurs intègrent ensuite les leçons des critiques menées par Team $X$ au sein des Congrès Internationaux d'Architecture Moderne (CIAM) qui conduisent à des propositions fondées sur l'idée de continuité urbaine (Bagnols-sur-Cèze, la Viste et La Maurelette à

6. B. Blanchon, «Les paysagistes français de 1945 à 1975, l'ouverture des espaces urbains ", Les Annales de la recherche urbaine $n^{\circ} 85$, Plan Urbanisme Construction Architecture, décembre 1999, p. 20-29 et «Pratiques et compétences paysagistes dans les grands ensembles d'habitations, 1945-1975», Strates n 13, Hélène Jannière et Frédéric Pousin (dir.), Ladyss / CNRS, 2007 ، p. 149-167. 

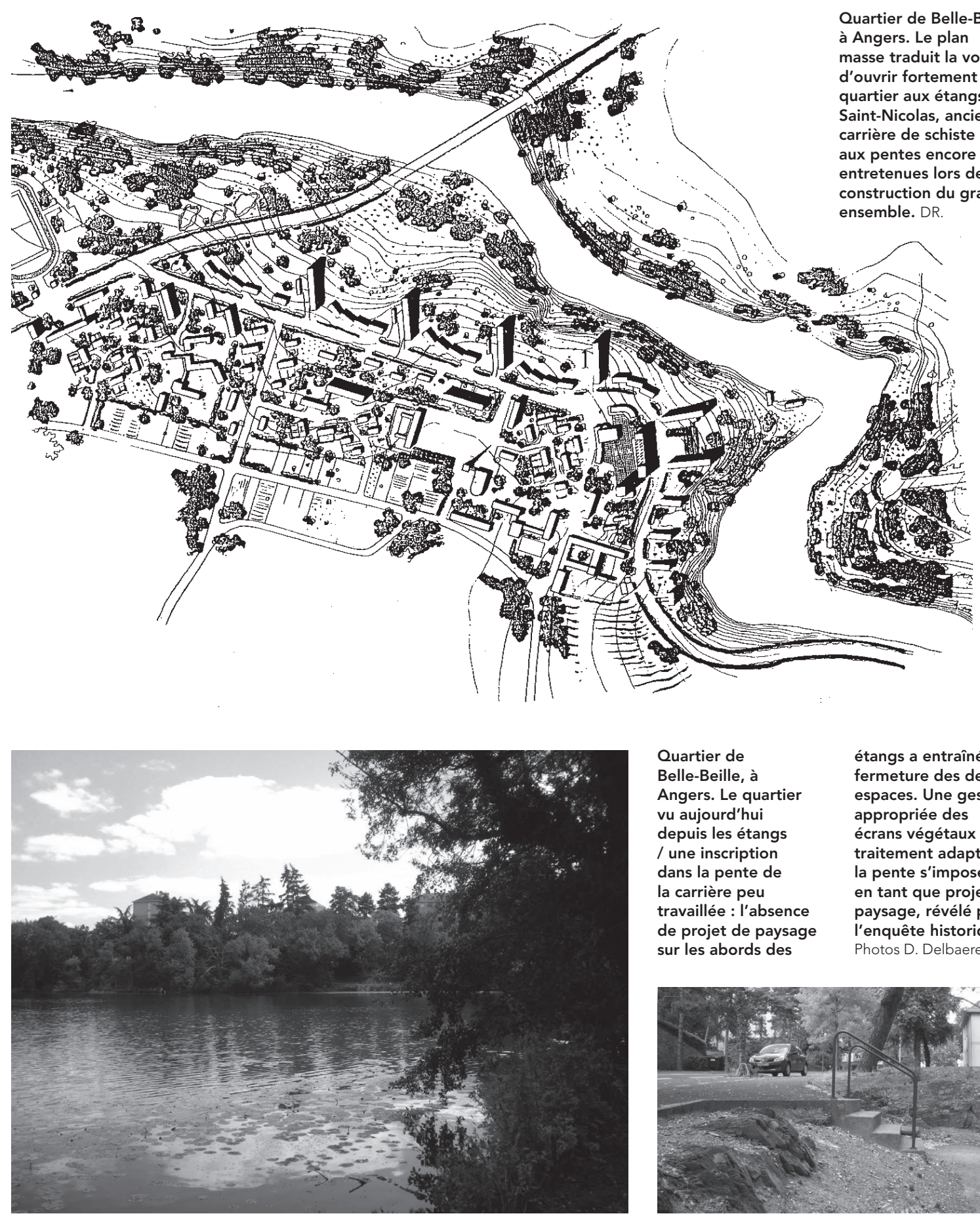

Quartier de

Belle-Beille, à

Angers. Le quartier

vu aujourd'hui

depuis les étangs

/ une inscription

dans la pente de

la carrière peu

travaillée : I'absence

de projet de paysage

sur les abords des étangs a entraîné la fermeture des deux espaces. Une gestion appropriée des écrans végétaux et un traitement adapté de la pente s'imposent en tant que projet de paysage, révélé par I'enquête historique. Photos D. Delbaere.

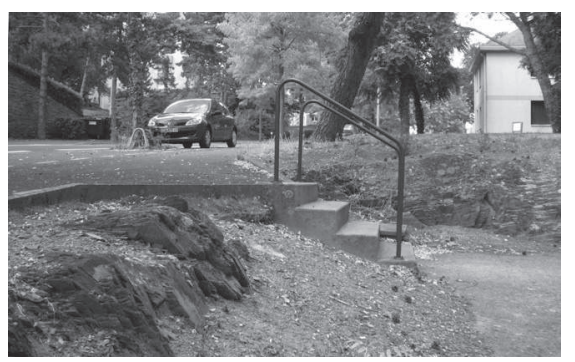


Marseille...) puis à des expérimentations sur les rues intérieures et les coursives (Le Mirail à Toulouse, La Villeneuve à Grenoble) mais aussi en grappes ou nappes (la Maladrerie à Aubervilliers, Givors, les Poètes à Pierrefitte en Seine-Saint-Denis...). En parallèle, plusieurs approches atypiques s'ajoutent aux précédentes : les approches plastiques d'Émile Aillaud, la soumission aux règles classiques de Fernand Pouillon et la recherche typologique et environnementale (paysage urbain, Townscape) menée par Robert Auzelle.

Mais cette approche "par le plan » passe pour partie à côté de la recherche de paysage que nous avons opérée, car si les plans de masse excellent à révéler comment les architectes ont sculpté l'espace ouvert, ils ne nous apprennent pas pour autant comment ils sculptent le paysage. Le paysage, en effet, se joue au sol et depuis le sol. Par un travail de terrain soutenu, nous l'avons apprécié par les parcours qui le traversent, par les modalités de passage d'un immeuble à un autre, d'un niveau à un autre, à travers le dialogue que l'opération entretient avec ses limites, et tout ceci aussi bien horizontalement par la distribution en plan des espaces, que verticalement par ce que le travail de terrassement instaure entre l'opération et son sol. Nos relevés ont porté particulièrement sur quatre composantes du paysage, également susceptibles de révéler le projet implicite ou explicite qui en est l'origine.

Premièrement, la maîtrise du matériau végétal, qui déploie toute une gamme de transparences et de modulations lumineuses. Très présente dans cette catégorie, la figure de l'allée plantée, déclinable du mail à la coulée verte, conçue comme outil de mise en relation fluide, continue, des espaces ouverts les uns avec les autres de manière autonome par rapport au plan de masse, révèle de nombreux projets, surtout dès qu'elle s'affranchit du strict accompagnement de la voirie : à la cité de l'Abreuvoir à Bobigny, (architecte, Émile Aillaud), il est l'armature même du projet, alors qu'à la Villeneuve de Grenoble (architecte, Atelier d'urbanisme et d'architecture AUA) il ressort de la simple évocation de figures traditionnelles du paysage cultivé. En coupe, ce mode relationnel peut se traduire par la dissociation du niveau des promenades d'avec celui des immeubles d'une part, de la voirie d'autre part. De nombreuses figures de filtres végétaux participent également de cette catégorie.

Deuxièmement, le jeu des masses bâties dans sa capacité à introduire et à relier les espaces ouverts du quartier. II comprend les figures de I'angle ouvert, du porche, de la coulisse. En coupe, seuils, terrasses et belvédères peuvent renforcer ces gammes subtiles de passages et de glissement d'un espace sur l'autre. À La Maurelette (Marseille), les bâtiments suivent le principe de continuité urbaine (inspiré par les tenants du Team X) au long d'un enchaînement de placettes minérales, reliées par des passages qui reprennent les modes de l'architecture locale. Ailleurs (Bas-Coudray à Sceaux), une double épaisseur de bâtiments négocie l'articulation de l'espace central avec les différents types de contextes périphériques...

Troisièmement, l'exploration des modes de nivellement, naturels ou artificiels, nous a offert une piste fructueuse dans notre recherche de paysages. Le terrassement structure le socle de l'opération, directement associé à l'architecture des rez-de-chaussée et des pieds 
de bâtiments, espaces d'entre-deux souvent sources de conflits aujourd'hui. De l'inscription dans une topographie naturelle (la Butte rouge, Beaulieu, Firminy...) à sa transformation radicale (la Villeneuve) en passant par le modelage fin du terrain au Village Olympique (Grenoble) où le quartier est surélevé par rapport au terrain naturel, comme à Marly Les Grandes Terres, pour intégrer des parkings, le dessin du sol, également support des cheminements, le terrassement peut être vu comme l'assise d'un projet de paysage implicite dans le site, intégrant parfois la gestion de la qualité du substrat et des eaux de ruissellement.
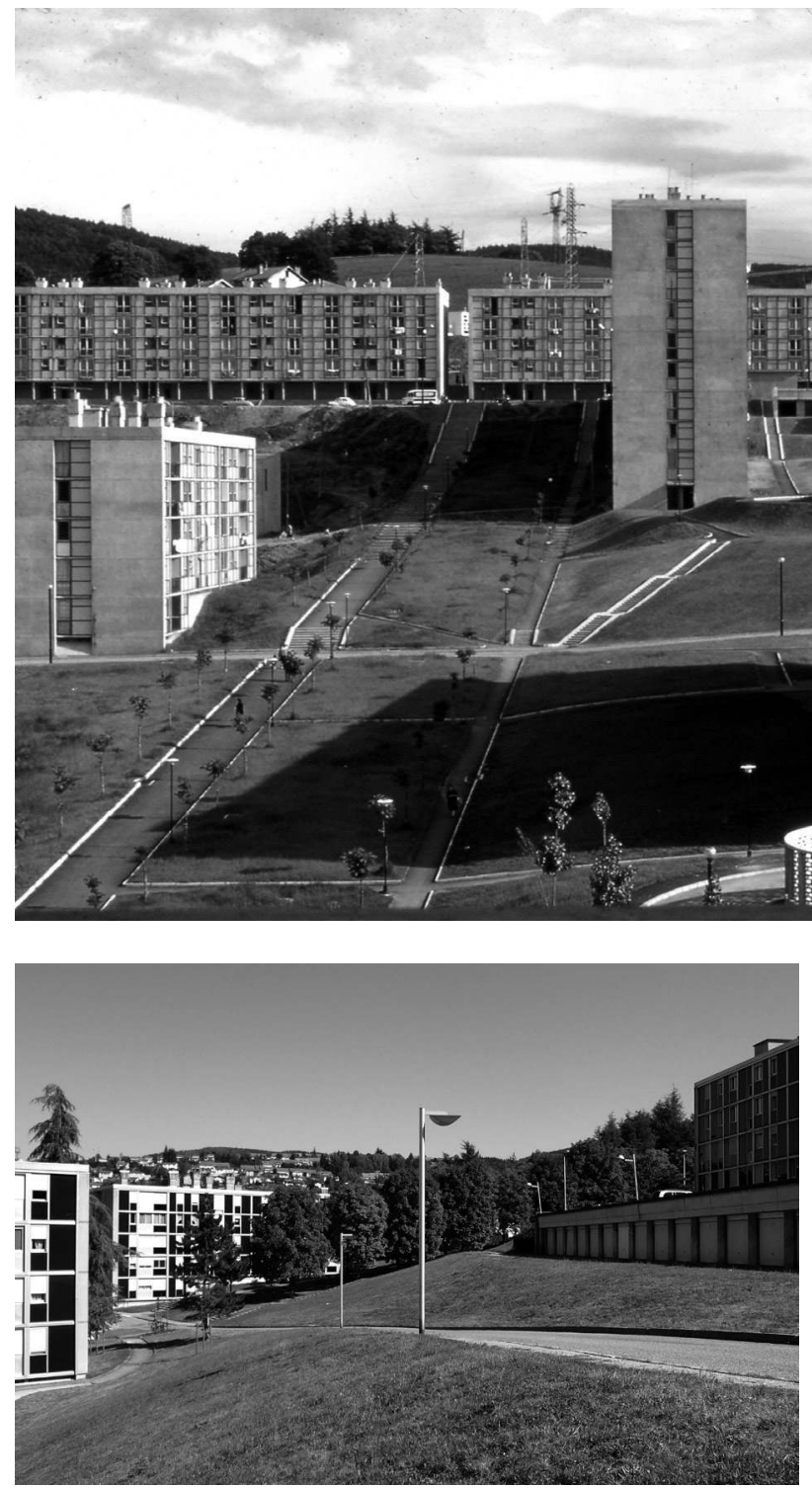

Firminy Vert : plus que des bâtiments remarquables conçus par Le Corbusier, le patrimoine exceptionnel présent ici est celui d'une ville moderne entièrement dessinée avec son paysage à toutes les échelles. La composition installe une cohérence lumineuse entre le site, la structure des
"Firminy-Vert " (urbaniste

Charles Delfante). Vue partielle du cours des Marronniers et du boulevard de la Corniche, depuis la Tour d'André Sive et Marcel Roux. Cliché non daté (1961-1965 ?). Photographe Eugène Claudius Petit (1907-1989).

Archives

municipales de Firminy, dossier ZPPAUP de FirminyVert 2006 et centre de documentation de la DRAC Rhône-Alpes. espaces ouverts et les dispositifs d'insertion dans la pente. Derrière les talus et plateformes on voit le mail des marronniers. À l'origine, un socle et une promesse végétale. Les bâtiments ne sont plus qu'un élément parmi d'autres dans un ensemble rassemblé par une logique paysagère. Photo B. Blanchon. 
Enfin, il nous est vite apparu que la tendance du parti général d'organisation des espaces ouverts à adresser le quartier à ses propres limites constitue également un marqueur de projet de paysage. En effet, penser le paysage, c'est penser le dedans par le dehors et la ville par son site. C'était d'ailleurs le propos de nombreux architectes modernes du fait des situations spécifiques auxquelles ils se trouvaient confrontés : la plupart des grands ensembles, parce qu'ils investissaient des terrains en périphérie urbaine, éloignés des fonds de vallée où les centres urbains se sont historiquement implantés, sont situés sur les coteaux pentus de ces vallées, si bien que leurs concepteurs pouvaient tirer parti facilement des effets de belvédère offerts par la coupe du terrain. Des projets comme le Haut du Lièvre à Nancy, sont emblématiques de l'obsession des architectes $d^{\prime}$ orienter les logements vers la lumière et le paysage lointain. Mais cette relation était généralement purement visuelle, et a produit souvent des non-sens complets. Les bâtiments du Haut du Lièvre, implantés en haut d'un plateau dominant la ville, s'interrompent au bord d'une falaise dont l'architecte n'avait pas prévu qu'en se boisant (du fait de la déprise agricole résultant de I'urbanisation elle-même), elle deviendrait un mur infranchissable par la vue comme par la marche. De nombreux projets magnifiquement composés dans la pente (Belle-Beille à Angers, le Shape Village à SaintGermain-en-Laye) présentent les mêmes " contresens paysagers ", où le végétal aujourd'hui referme les horizons au lieu de les accompagner. Penser le paysage dans ces quartiers aurait consisté donc à aménager au-delà du périmètre des travaux, à imaginer le devenir des franges. Afin de ne pas alourdir le budget des opérations, des modes de gestion et d'aménagement élémentaires auraient suffi à garantir la perméabilité physique et visuelle qui y fait aujourd'hui si souvent défaut. Cette problématique n'a pas échappé aux paysagistes, et Jacques Simon, dans ses chroniques de la revue Espaces verts, s'est fait, à partir de 1969, le témoin critique de cette tendance au repli due à un défaut de pensée sur les marges ${ }^{7}$. Pour lui, le dialogue entre le programme et le site devait être recherché là où les deux se rencontrent, c'est-à-dire aux limites du projet, pensées comme des espaces de confrontation, de porosité, d'échange, de transition, de seuil.

Ces dispositifs variés sont aujourd'hui d'usage courant dans les projets de paysage (et restent cependant peu connus des acteurs du projet urbain), mais c'est à cette époque qu'il a été possible de les tester. L'évolution des projets entre 1940 et 1980 montre comment ces espaces de marge sont devenus progressivement le véritable centre du projet. Peu à peu, les formes matérielles des espaces libres se complexifient: elles convoquent les figures locales de l'espace public, comme à la Maurelette où le registre provençal est largement sollicité. À l'intérieur des plans de masse, les mails prennent de la largeur et de la longueur, et tentent d'ouvrir des perspectives vers un ailleurs. Surtout, les parcs s'éloignent du centre du quartier, pour s'implanter en périphérie, et former contact avec la campagne ou la ville alentour. Des espaces à valeur de manifeste comme le parc Saint-John Perse en périphérie du quartier de la Croix Rouge à Reims, le parc de la Courneuve, ou plus tard, le parc du Sausset en bordure du programme

7. Frédéric Pousin (dir.), Saisir le paysage urbain, rapport de recherche, Programme interdisciplinaire de recherche « Art, architecture, paysage », ministère de la Culture et de la Communication, BRAUP, 2007. 
Aulnay 3000, manifestent cet excentrement progressif de l'espace paysager.

Plus que la lecture en plan, c'est donc le repérage à partir du terrain de ces dispositifs-témoins, de ces marqueurs de projet de paysage (même quand aucun concepteur paysagiste n'est identifié) qui a guidé notre travail et nous a permis de lire un projet à rebours là où les sources archivistiques l'abandonnent au blanc de la carte.

\section{Conclusion}

\section{Projeter à rebours pour relancer}

\section{le processus du projet de paysage}

Au terme de cette recherche, une centaine de grands ensembles, soit plus du tiers des espaces investigués, fait l'objet d'un signalement à l'attention des préfets et des services déconcentrés du ministère de la Culture et de la Communication, afin de veiller à ce que les qualités relevées puissent être prises en compte lors du remodelage des quartiers. La balle est désormais dans le camp des préfets qui, région par région, auront à faire valoir cet héritage moderne ! La dynamique de projet, fondée sur une valorisation prospective parce que rétrospective du paysage, est engagée.

Mais encore? Quelle forme pourra-t-elle prendre? La réponse la plus littérale suggérerait la mise en place de mesures conservatoires pour les espaces de qualité paysagère mis en évidence. Nous espérons cependant que cette prise en compte ne prendra pas la forme d'une mise sous globe de quelques figures fortes du "patrimoine " paysager de ces quartiers, mais s'attachera davantage à renouer avec les logiques structurelles qui ont présidé à leur création. Ce serait effectivement un contresens que de raisonner en termes de formes, alors que pour lire ces formes, il a justement fallu ne pas s'en tenir aux formes présentes sur le terrain, mais les penser dans le cadre élargi d'un contexte géographique, territorial, et d'une continuité spatiale expérimentée lors de nos explorations sur le terrain. Le projet de paysage n'entretient pas la même relation à l'aboutissement formel que le projet architectural. Ici, ni la forme, ni le matériau, ni les usages qui les mobilisent ne sont pleinement maîtrisables. La réussite d'un projet de paysage est moins affaire de forme que de pertinence, c'est-à-dire de capacité à s'inscrire, en la confortant et en la rendant profitable pour le plus grand nombre, dans une dynamique de transformation de l'espace qui excède largement la durée du seul segment de la conception et de la maîtrise d'œuvre. Pour cette raison, lorsque le chercheur investit un terrain et tâche, par la lecture de sa géographie, de ses espaces et des formes qui les structurent, de le saisir comme un ensemble cohérent, il fait acte de projet au même titre, mais de manière inverse, que le concepteur chargé de le dessiner. Le second projette le paysage comme ce qui doit advenir en s'appuyant sur ce qui est d'ores et déjà là. Le premier le révèle comme ce qui nous est donné, en s'appuyant sur ce qu'on a voulu en faire. 